Iron metabolism and Hallervorden-Spatz syndrome are reviewed in ped Neur Briefs October 2001;15:75-76.

Genetics of febrile seizures. A splice-site mutation in the GABRG2 gene has been described that causes a nonfunctional truncation of the GABA receptor $\mathrm{g}$ subunit, and contributes susceptibility to childhood absence epilepsy and febrile convulsions in a single family. (Kananura C, Haug K, Sander T et al. Arch Neurol July 2002;59:1137-1141). The association of a missense mutation in the GABRG2 gene and susceptibility to febrile seizures is a rare finding, previously reported and cited by the authors in only one other family (Wallace RH et al. Nat Genet 2001;28:49-52).

\title{
VISUAL ATTENTION IN WEST SYNDROME
}

The maturation of visual attention is evaluated prospectively in a study of infants with West syndrome (WS) before, during and after the onset of seizures, followed until age $24+/-2$ months at Catholic University, Rome, and University of Pisa, Italy. Thirteen infants with symptomatic WS and 22 normal control infants received a serial complete assessment at age 3 months, at the time of onset of spasms, and at 2 years. Visual attention assessment was clinical and also by means of a fixation-shift system, the ocular behavior monitored by video recordings. The median onset of spasms was 7.5 months (range, 3.5-12 months). EEG showed bilateral epileptic discharges, dominant in the occipital regions in 5; 7 had typical and 6 a modified hypsarrhythmia. Before onset of spasms, fixation and tracking were present in all infants, but only $4(30 \%)$ could pass the fixation-shift test. At onset of spasms, the majority (70\%) showed severe impairment of visual behavior; only $4(30 \%)$ responded to fixation and tracking, and only one responded correctly to the fixation-shift test. At age 2 years, a general improvement in visual attention had occurred; fixation and tracking were possible in all cases, but only 2 could perform the fixation-shift test correctly. Cognitive development (DQ) paralleled the visual maturation: borderline DQ before onset of spasms, a general deterioration to a median score of 35 at onset of spasms, with minor improvement at age 2 year follow-up. In 2 cases with normal DQ at 3 months, a parallel deterioration of fixation-shift skills and cognitive development was observed even some months before onset of spasms. (Guzzetta F, Frisone MF, Ricci D, Rando T, Guzzetta A. Development of visual attention in West syndrome. Epilepsia July 2002;43:757-763). (Reprints: Dr F Guzzetta, Neuropsichiatria Infantile, Policlinico Gemelli, UCSC, Largo Gemelli 8, 1-00168 Rome, Italy).

COMMENT. A parallel defect of visual attention and cognitive development occurs in infants with West syndrome and sometimes precedes the onset of infantile spasms. In an infant with delayed development who presents with visual inattention, a diagnosis of West syndrome should be considered in the differential diagnosis and an EEG performed as part of the evaluation. The occipital preponderance of hypsarrhythia frequently reported in cases of WS appears to correspond to the common occurrence of visual inattention in this syndrome.

\section{TOPIRAMATE AND METABOLIC ACIDOSIS IN INFANTS}

The acid-base metabolism was investigated in 9 infants and toddlers, aged 5 months to 2.3 years (median, 6 months), treated with topiramate (TPM) for seizures at Johannes Gutenberg University, Mainz, Germany. TPM was used in maximal doses of $8.2-26 \mathrm{mg} / \mathrm{kg} /$ day (median, $11 \mathrm{mg} / \mathrm{kg} /$ day), as add-on therapy in 5 and monotherapy in 4 patients with refractory seizures. Diagnoses were infantile spasms in 5, epilepsia partialis continua in 1, infantile epileptic 
encephalopathy in 1, and Lennox-Gastaut syndrome in 2 cases. Metabolic acidosis developed in 8 patients after 8-26 days (median, 14 days) of TPM treatment. Median serum bicarbonate was $17 \mathrm{mM}$, minimal base excess $-7.9 \mathrm{mM}$, and pH between 7.22 and 7.40 (median, 7.35). Four children had clinical signs of hyperventilation and required oral sodium bicarbonate; TPM effectiveness was not affected. Monitoring of acid-base metabolism is recommended in young children during therapy with TPM. (Philippi H, Boor R, Reitter B. Topiramate and metabolic acidosis in infants and toddlers. Epilepsia July 2002;43:744-747). (Reprints: Dr med H Philippi, Department of Pediatrics, Johannes Gutenberg-University, Mainz, Germany).

COMMENT. Metabolic acidosis, a consequence of carbonic anhydrase inhibition, is a frequent side effect of topiramate in infants and toddlers during treatment for refractory seizures. Since the mechanism of anticonvulsant action of carbonic anhydrase inhibitors is unrelated to the metabolic acidosis (Millichap JG, Woodbury DM, Goodman LS. Mechanism of the anticonvulsant action of acetazolamide, a carbonic anhydrase inhibitor. I Pharmac Exp Therap $1955 ; 115: 251)$, the addition of sodium bicarbonate to TPM to correct hyperventilation would not be expected to lead to a recurrence of seizures.

\section{ATTENTION DEFICIT DISORDERS}

\section{NONSTIMULANT THERAPY FOR ADHD}

The results of a multicenter, open-label, dose-titration study of GW320659, a novel norepinephrine and dopamine reuptake inhibitor, are reported from GlaxoSmithKline, Research Triangle Park, NC. Forty six subjects with ADHD, mean age 9.1 years, received the maximal acceptable dose (mean $14.2 \mathrm{mg} /$ day) continued for a 4-week treatment period. During the initial dose-titration period, a clinical response was judged by a Clinical Global Impression of Improvement score of 1 or 2 , and a 5 or more point improvement on at least one Conners Rating Scale T score. At the end of treatment, $76 \%$ of subjects showed improvements; 7 of 12 subscales of the Child Health Questionnaire Parent Form 28 showed significant improvements compared with baseline $(\mathrm{p}<.05)$. Adverse events reported in $92 \%$ of subjects included headaches in $31 \%$, abdominal discomfort (25\%), excessive crying (20\%), anorexia (18\%), insomnia (14\%), and nausea and vomiting (12\%). Five subjects (10\%) required reduction in dose during the titration phase because of adverse events: these included crying and emotional lability in 2, mood elevation (1), increased blood pressure (1), and sleep disorder and nocturia (1). No serious side effects occurred that necessitated drug withdrawal. The authors concluded that GW320659 may have "clinically relevant efficacy" in pediatric ADHD and was "well tolerated" in this short-term study. (DeVeaugh-Geiss J, Conners CK, Sarkis EH et al. GW320659 for the treatment of attention-deficit/hyperactivity disorder in children. LAm Acad Child Adolesc Psychiatry August 2002;41:914-920). (Reprints: Dr Asgharnejad, GlaxoSmithKline, Five Moore Drive, PO Box 13398, Research Triangle Park, NC 27709).

COMMENT. Stimulant medications such as methylphenidate and amphetamines are the first line of treatment in most children with ADHD. Alternative treatments such as clonidine and guanfacine have indications for patients with comorbid ODD, insomnia, and tics, and bupropion is sometimes recommended in ADHD patients, especially those who exhibit depressive symptoms. GW320-6590, like bupropion, inhibits norepinephrine and dopamine reuptake. Both stimulants and bupropion may exacerbate tics and increase susceptibility to seizures, side effects not reported in the trial of GW320-6590. 